\title{
Progressive and Controllable Fractal Image Decoding Algorithm
}

\author{
Zong-wen Yuan, Ye-pin Lu, Xin-he Chen \\ College of Electronic Engineering \& Electricial Automation, Chaohu College, Chaohu 238000, China
}

Keywords: Fractal; Decoding; Compression mapping; Fixed point; Convergence; Progressive

\begin{abstract}
Although faster image decoding method is more important and useful, in some special applications, the controllable decoding process may be required. In the case of making fixed point unchanged, the paper made the fixed parameters of compression mapping into variable parameters, adjusted these parameters to make process controllable. Experimental results showed that the traditional fractal image decoding required about 8 times iteration to convergence,in contrast to requiring about 36 times iteration to convergence in this paper. The decoding process could be flexibly controlled, and the algorithm could better show the details of the decoded image sequence, compared with the literature [4].
\end{abstract}

\section{Introduction}

In the past two decades, scholars have published a number of papers ${ }^{[1-2]}$, mostly around how to accelerate the speed of fractal image coding; in addition, although the fractal decoding is fast enough, there are still few papers discuss how to accelerate the speed of the fractal decoding ${ }^{[3]}$. However, in some special applications, such as computer-aided production of cartoons, teaching of image decoding and the narrowband transmission of signal, it needs a slow, controllable and progressive decoding method. JPEG2000 supports progressive image transmission technology, which solves the image information transmission with progressive method in the narrow-band channels, that is, transmitting the contour information of the image with lower stream at first, and then use the same lower stream to gradually transmit the detailed information of the image. This not only can solve the anxious waiting problem of narrowband users, but also can meet the different requirements of users on the image quality. However, the fractal image coding has not been applied on progressive transmission, and the key reason is the traditional fractal decoding is uncontrollable, and the iteration time required from the initial image to the attractor is fixed, which are generally about seven to eight steps. The literature ${ }^{[4]}$ proposed a controllable fractal image decoding algorithms to better achieve the progressive decoding purposes. In this paper, it makes some improvements on the algorithms in this literature to control the decoding process more flexible and more uniformly.

\section{Algorithm Analysis}

\section{Fixed Point Theorem}

Theorem 1: Define the change $W: \psi \rightarrow \psi$ on the image space $(\psi,\|\cdot\|)$. This the existing of $0 \leq s<1$ makes

$$
\|(W(C), W(B))\| \leq s\|C, B\|, \quad \forall C, B \in \psi
$$

Then, $\boldsymbol{W}$ called compressed mapping. If the image sequence $\left\{\mathbf{U}_{n}\right\}$ meets $\boldsymbol{U}_{n+1}=\boldsymbol{W}\left(\boldsymbol{U}_{n}\right), n \geq 0, \boldsymbol{U}_{0}=\boldsymbol{U}$, $\boldsymbol{U}$ is an arbitrary image in the space $(\psi,\|\cdot\|)$,then the image sequence has the only fixed point $\mathbf{U}^{*}=\mathbf{U}_{\infty}$ under the effect of compressed mapping $\boldsymbol{W}$, that is to say, it exists the only image $\boldsymbol{U}^{*}$ to make $W\left(U^{*}\right)=U^{*}$.

Theorem 2: Define the change $S: \psi \rightarrow \psi$ on the image space $(\psi,\|\cdot\|)$, it meets

$$
\boldsymbol{S}\left(\boldsymbol{U}_{n}\right)=w_{1} \boldsymbol{U}_{n}+w_{2} \boldsymbol{W}\left(\boldsymbol{U}_{n}\right)+w_{3} \boldsymbol{W}^{2}\left(\boldsymbol{U}_{n}\right)
$$


In for formula, weighting coefficient $w_{1}, w_{2}, w_{3} \in[0,1)$, and $w_{1}+w_{2}+w_{3}=1, W$ is the compressed mapping in the theorem 1, then $S$ belongs to the compressed mapping and with the same fixed point of $W$.

$$
\begin{aligned}
& \text { Proof: For the arbitrary } \boldsymbol{U}, \boldsymbol{V} \in \boldsymbol{\psi} \text {, there is } \\
& \|\boldsymbol{S}(\boldsymbol{U})-\boldsymbol{S}(\boldsymbol{V})\|=\| w_{1}(\boldsymbol{U}-\boldsymbol{V})+w_{2}(\boldsymbol{W}(\boldsymbol{U})-\boldsymbol{W}(\boldsymbol{V}))+ \\
& w_{3}\left(\boldsymbol{W}^{2}(\boldsymbol{U})-\boldsymbol{W}^{2}(\boldsymbol{V})\right) \| \\
& \leq\left\|w_{1}(\boldsymbol{U}-\boldsymbol{V})\right\|+\left\|w_{2}(\boldsymbol{W}(\boldsymbol{U})-\boldsymbol{W}(\boldsymbol{V}))\right\|+ \\
& \left\|w_{3}\left(\boldsymbol{W}^{2}(\boldsymbol{U})-\boldsymbol{W}^{2}(\boldsymbol{V})\right)\right\| \\
& \leq w_{1}\|\boldsymbol{U}-\boldsymbol{V}\|+s w_{2}\|\boldsymbol{U}-\boldsymbol{V}\|+s w_{3}\|\boldsymbol{W}(\boldsymbol{U})-\boldsymbol{W}(\boldsymbol{V})\| \\
& \leq w_{1}\|\boldsymbol{U}-\boldsymbol{V}\|+s w_{2}\|\boldsymbol{U}-\boldsymbol{V}\|+s^{2} w_{3}\|\boldsymbol{U}-\boldsymbol{V}\| \\
& =\left(w_{1}+s w_{2}+s^{2} w_{3}\right)\|\boldsymbol{U}-\boldsymbol{V}\|
\end{aligned}
$$

Because $0 \leq s<1$, so $0 \leq w_{1}+s w_{2}+s^{2} w_{3}<1$, so $S$ belongs to the compressed mapping. On the other hand, if $\boldsymbol{U}^{*}$ is the fixed point of $\boldsymbol{W}$, then $\boldsymbol{S}\left(\boldsymbol{U}^{*}\right)=w_{1} \boldsymbol{U}^{*}+w_{2} \boldsymbol{W}\left(\boldsymbol{U}^{*}\right)+w_{3} \boldsymbol{W}\left(\boldsymbol{W}\left(\boldsymbol{U}^{*}\right)\right)$

$$
=w_{1} \boldsymbol{U}^{*}+w_{2} \boldsymbol{U}^{*}+w_{3} \boldsymbol{U}^{*}=\boldsymbol{U}^{*}
$$

Therefore, $\boldsymbol{U}^{*}$ is also the fixed point of S. So, S belongs to the compressed mapping and with the same fixed point of $W$.

Theorem 2 has completed the proof.

\section{Progressive and Controllable Fractal Decoding}

The traditional fractal decoding is the compressed mapping $\boldsymbol{W}$ described by the fractal codes completed by the repeated iteration towards one arbitrary image $\boldsymbol{U}$, and according to the collage theorem, the fixed point $\boldsymbol{U}^{*}$ us an approximate image of the original image $\boldsymbol{U}_{\text {org }}$. That is:

$$
\boldsymbol{U}_{\text {org }} \approx \boldsymbol{U}^{*}=\lim _{n \rightarrow \infty} \boldsymbol{W}^{n}(\boldsymbol{U}) \approx \boldsymbol{W}^{N}(\boldsymbol{U}), \quad \forall \boldsymbol{U} \in \boldsymbol{\psi}
$$

Typically, no matter what the original image is, the $N$ satisfied the equation is approximately 8 , so this is an iterative process can not be controlled. Based on Theorem 2, we use the compressed mapping $\boldsymbol{S}$ to replace $\boldsymbol{W}$, and through changing weighting coefficient, it can realize the control of the iterative process, and its fixed point is an approximate image of the original image, that is, the quality of final decoded image is the same. The implemented program is as described in Fig 1 (b).

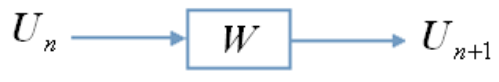

(a)

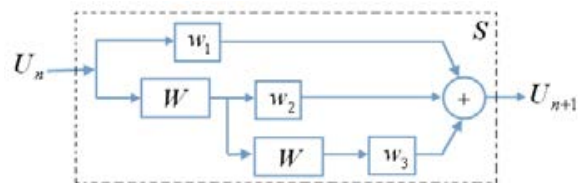

(b)

Fig.1 Fractal Decoding: (a) Traditional Decoding Program; (b) Progressive Controllable Decoding Program

In Fig. 1 (b), when $w_{3}$ equals to zero, this program changes into the progressive decoding program proposed from the literature [4], so the program from the literature is just a special case of this program. Of course, on this basis, it can construct a more complex progressive decoding program, for example, it can construct to change 


$$
\boldsymbol{S}\left(\boldsymbol{U}_{n}\right)=w_{1} \boldsymbol{U}_{n}+w_{2} \boldsymbol{W}\left(\boldsymbol{U}_{n}\right)+w_{3} \boldsymbol{W}^{2}\left(\boldsymbol{U}_{n}\right)+\cdots+w_{N} \boldsymbol{W}^{N-1}\left(\boldsymbol{U}_{n}\right)
$$

In the equation, the weighting coefficients $w_{1}, w_{2}, w_{3}, \cdots, w_{N} \in[0,1)$, and $w_{1}+w_{2}+w_{3}+\cdots+w_{N}=1$, can prove that this $\mathrm{S}$ also belongs to the compressed mapping, and has the fixed point similar to $W$. However, it can be expected that such decoding program can reduce its usefulness due to its complexity. In addition, in this program, due to it contains twice compressed mapping, so when the time of pre-set iterations $N$ is an odd number, the former $(N-1) / 2$ time adopts this program. And for the last time, which is the $(N-1) / 2+1$ time, it can make $w_{3}=0, w_{2}=1-w_{1}$, while $w_{1}$ remains unchanged, then conducts the iterative decoding, so it can arbitrarily pre-set the number of iterations, while ensuring the fixed point of the image unchanged.

\section{Analysis of Experimental Results}

In the weighting coefficients $w_{1}, w_{2}, w_{3}$ of this program, there are only two variables are independent, and we can arbitrarily change two coefficients to achieve controlling the decoding speed. In order to show the impact of the coefficient towards decoding, in the experiments, we take a $512 \times 512$ gray image of Lena, use the traditional program of decoding, use the fixed segmentation of the image, and the size of range block is $8 \times 8$, so when decoding, $w_{2}$ and $w_{3}$ take different values, the progressive decoding result obtained is as shown in Fig. 2.

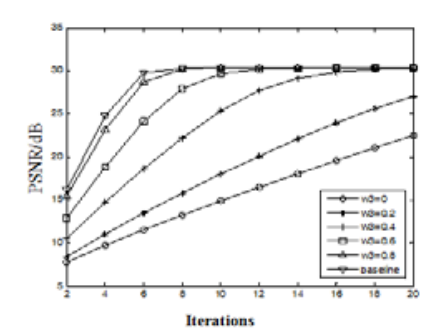

(a) $w_{2}=0.2$

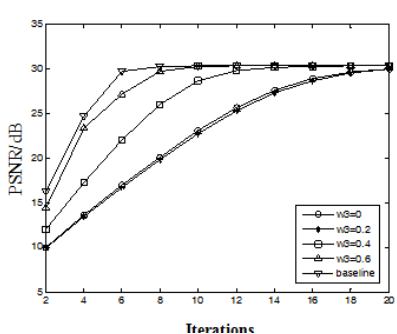

(b) $w_{2}=0.4$

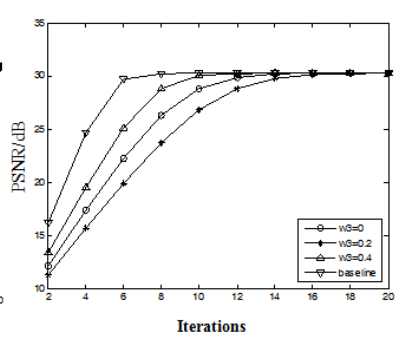

(c) $w_{2}=0.6$

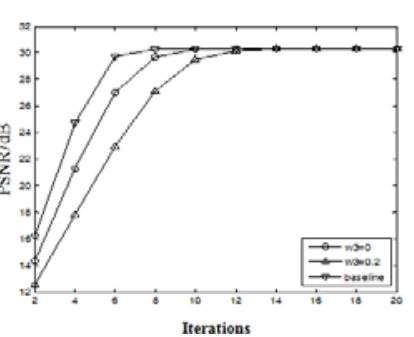

(d) $w_{2}=0.8$

Fig.2 Decoding Results when ${ }^{w_{2}}$ and ${ }^{w_{3}}$ Take Different Value

In Fig.2, in the graphic symbol, the baseline curve represents the traditional fractal decoding, $w_{3}=0$ curve is the decoding of the literature [4], and other curve is the decoding of this program. It can be seen that the speed of the decoding in this program is slower than that of the traditional decoding, and the decoding in this program has more options and its decoding is controllable. In order to subjectively feel the progressive decoding character is this program, we can choose any of a non-baseline curve from Fig.2, and show its decoding process by using the sequence of images. For example, take the corresponding curves of $w_{2}=0.2, w_{3}=0.2$, it obtains the sequence of decoded images is shown in Fig. 3. The initial image is a black image, and the Fig. $3(\mathrm{k})$ is the $36^{\text {th }}$ iteration, in which PSNR $=30.26 \mathrm{~dB}$, and has $0.05 \mathrm{~dB}$ difference between the PSNR $=30.31 \mathrm{~dB}$ of the fixed image, indicating that it is basically converged. (See Fig.2 (a)) 

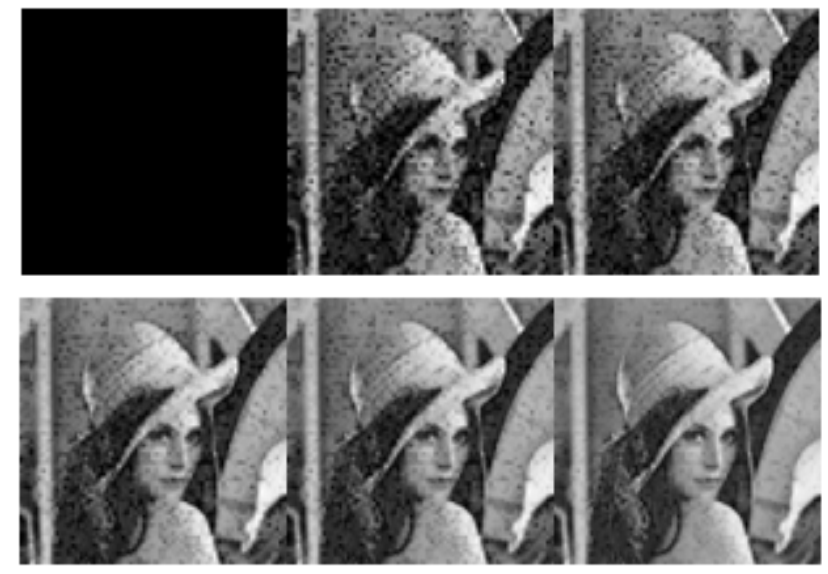
(a) Initial Image
(b) 2nd Iteration
(c) 4th Iteration
(d) 6th Iteration
(e) 8th Iteration
(f) 10th

Iteration
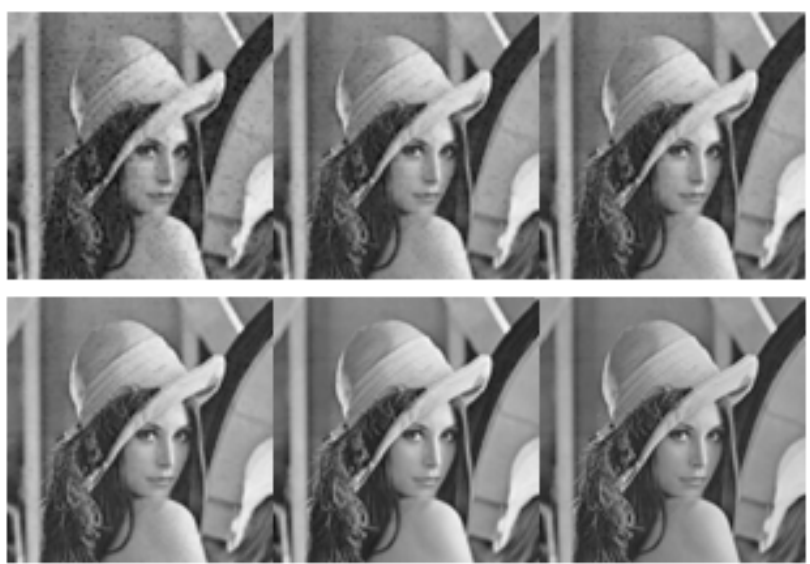
(g) 12th Iteration
(h) 14th Iteration
(i) 16th Iteration
(j) 18th Iteration
(k) 36th Iteration
(l) Original Image

Fig. 3 Sequence of the Decoded Image in This Program $\left(w_{2}=0.2, w_{3}=0.2\right.$ )

For comparison, take the baseline curve from Fig.2 to show the decoding process, that is, the process of traditional fractal decoding, and the resulting sequence of image is shown in Fig.4. Fig.4 (e) is the $8^{\text {th }}$ iteration, and its PSNR $=30.28 \mathrm{~dB}$, showing it is basically converged. It can be seen that the convergence speed of decoding in this program is slower than that of the traditional decoding, while if adjusting the weighting coefficients, it can achieve the different convergence speeds, especially, if $w_{2}=1$, this program will become the traditional program.

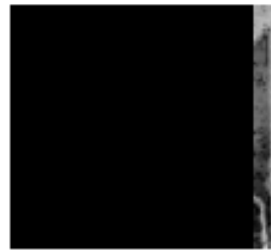

(a) Initial Image

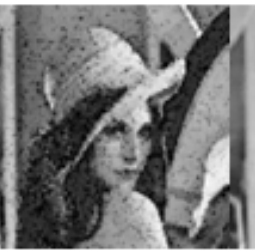

(b) 2nd Iteration

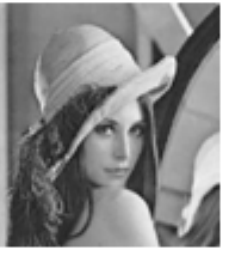

(c) 4th Iteration

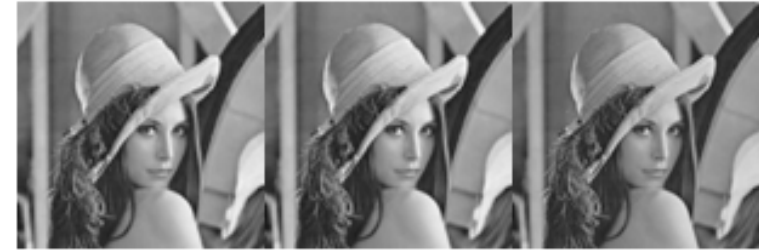

(d) 6th Iteration

(e) 8th Iteration

(f) Original

Fig.4 Sequence of Decoded Image in the Traditional Program ( $w_{2}=1$ )

Although both this program and the program in the literature [4] have the characteristics of progressive and controllable decoding, the control in this program can be more flexible and changeable. In order to illustrate this characteristic, we calculate the difference image of the neighbored decoding image to show the increase of its details. If $\boldsymbol{U}_{n}$ represents the decoded image of the $\mathrm{n}^{\text {th }}$ iteration. For Fig.5 and Fig.6, from left to right and from top to bottom, they 
are $\boldsymbol{U}_{2}-\boldsymbol{U}_{0}, \boldsymbol{U}_{4}-\boldsymbol{U}_{2}, \boldsymbol{U}_{6}-\boldsymbol{U}_{4}, \ldots \boldsymbol{U}_{18}-\boldsymbol{U}_{16}$. The results show that with the increase of the number of iterations, the details of the image in this program increase with a relatively uniform proportion, while for the program in the literature [4], we have conducted a large number of testing, and find that no matter how the coefficient changes, the change process of its difference image is shown in Fig.6 (Due to the limitation of space, we are inconvenient to show more results, but just take a set from them, such as $\left.w_{2}=0.4, w_{3}=0\right)$. It can be seen that the details of the image in Fig. 6 will no longer increase in the relatively flat area; however, the convergence speeds of other details are more quickly. Therefore, for some potential applications, this program may be more favorable, and in this program, if we change the coefficient, it can get more features of changes.

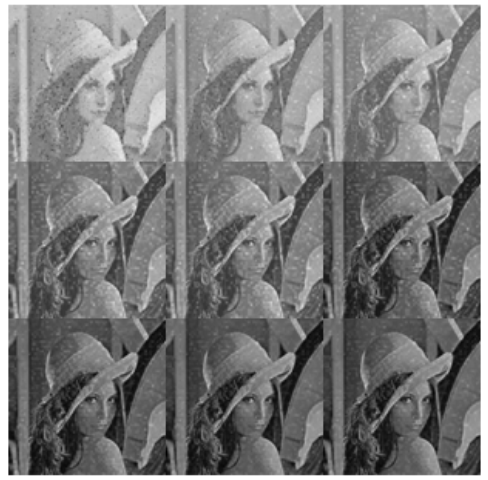

Fig.5 Difference Image ( $w_{2}=0.2, w_{3}=0.2$ )

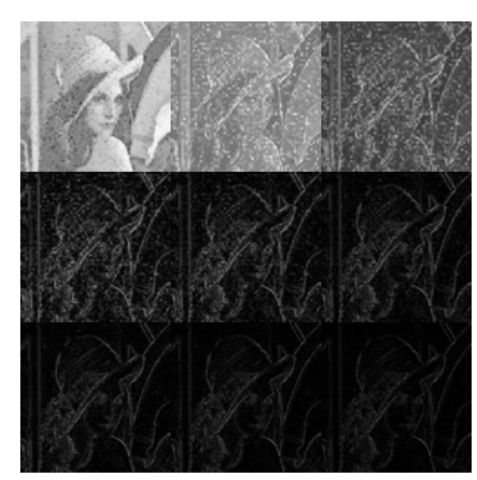

Fig.6 Difference Image ( $w_{2}=0.4, w_{3}=0$ )

Fig. 5 and Fig. 6 only show the differences of subjective quality between the decoding image sequences in two programs. In order to further subjectively analyze the characteristics of this program, we conduct the tests on the Lena and Baboon image in different degree of complexity, and give the PSNR curves of the neighbored difference image for two programs in the form of Fig.7 (The setting of coefficients are the same with Fig.5 and Fig.6). In Fig.7, the abscissa represents the sequence of difference images, such as, 8 corresponds to the difference image of $\boldsymbol{U}_{16}-\boldsymbol{U}_{14}$.

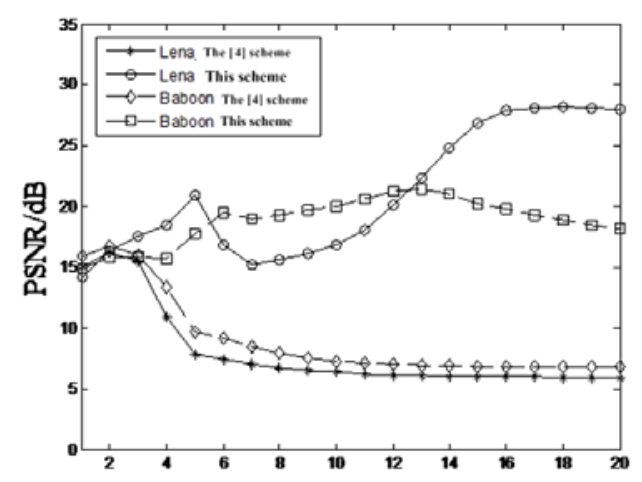

Fig.7 PSNR of Difference Image in Two Kinds of Decoding Program

From Fig.7, it can be seen that, for Lena and Baboon, the decoding difference image of this program have a higher PSNR, illustrating that in the whole decoding process, the increase of the image details in this program is more uniform, while the increase of the decoding image sequence details in the literature [4] approximates to the decay with the exponential law.

\section{Conclusion}

Based on the uncontrollable characteristic in the traditional fractal decoding process, it proposed a controllable and progressive fractal decoding algorithm, and this decoding algorithm does not change the original decoding algorithm, and the implementation process is simple. For some special applications, there are the potential application values. 
Experimental results show that the proposed algorithm not only has achieved a progressive decoding, but also has a more flexible, uniform and progressive controlling decoding performance compared to the algorithm in the literature [4].

This progressive and controllable algorithm currently is limited in the early theoretical research, so how to apply to engineering practice, to achieve the progressive transmission and progressive display of images, and what kind of disadvantages compared with the advanced decoding algorithm such as wavelet, still remain to be further studied.

\section{Acknowledgments}

Fund Program: Research Project of National Science in the Colleges and Universities in Anhui Province Fund Support, and Project Batch Number: KJ2011B103.

\section{References}

[1] Wohlberg B, Jager G D. A review of the fractal image coding literature. IEEE Transactions on Image Processing,1999,8(12): 1716-1729.

[2] Zhao Y, Wang $\mathrm{H}$ X, Yuan $\mathrm{B}$ Z.A dvanced in Fractal Image Coding. ACTA ELECTRONSINICA,2000,28(4):95-100.

[3] Ding Z J, Yuan Z W, Miao D S. Algorithm for Fast Fractal Image Decoding Based on Adjusted Contrast Factors . Journal of Lanzhou University of Technology, 2007, 33(4):110-112.

[4] He C J, Yang S X, Huang X Y. Progressive decoding method for fractal image compression. IEE Proceedings. Image and Signal Processing,2004,151(3):207-213. 